\title{
Performance Analysis of an Inverse Notch Filter and Its Application to $F_{0}$ Estimation
}

\author{
Yosuke Sugiura, Arata Kawamura, Youji Iiguni \\ Graduate School of Engineering Science, Osaka University, Osaka, Japan \\ Email: yosuke@sip.sys.es.osaka-u.ac.jp
}

Received November 21, 2012; revised December 21, 2012; accepted December 29, 2012

\begin{abstract}
In this paper, we analyze an inverse notch filter and present its application to $F_{0}$ (fundamental frequency) estimation. The inverse notch filter is a narrow band pass filter and it has an infinite impulse response. We derive the explicit forms for the impulse response and the sum of squared impulse response. Based on the analysis result, we derive a normalized inverse notch filter whose pass band area is identical to unit. As an application of the normalized inverse notch filter, we propose an $F_{0}$ estimation method for a musical sound. The $F_{0}$ estimation method is achieved by connecting the normalized inverse notch filters in parallel. Estimation results show that the proposed $F_{0}$ estimation method effectively detects $F_{0} s$ for piano sounds in a mid-range.
\end{abstract}

Keywords: Band Pass Filter; Inverse Notch Filter; Impulse Response Analysis

\section{Introduction}

In speech processing, image processing, biomedical signal processing, and many other signal processing fields, it is important to eliminate the narrowband signal. The examples of the narrowband signal are a hum noise from the power supply, an acoustic feedback, and an interfereence noise, and so on. A notch filter is useful for the elimination of the narrowband signal [1-7], where the notch filter passes all frequencies expect of a stop frequency band centered on a center frequency, called as the notch frequency. The notch filter has a simple structure, and its stop bandwidth and its notch frequency are individually designed. The notch filter is used in many applications and it has been analyzed in many literatures [1,4-7].

On the other hand, an inverse notch filter is a band pass filter which has the inverse characteristics of the notch filter. In contrast to the notch filter, there are few applications of the inverse notch filter. As an example of the applications, an active noise control system for reducing a sinusoidal noise has been proposed [8]. In this system, the inverse notch filter is used to extract the sinusoidal noise. Unfortunately, the system is designed without respect to the impulse response of the inverse notch filter. Hence, the inverse notch filter cannot accurately extract the sinusoidal noise when the filter output is in the transient state. To utilize the inverse notch filter more effectively for not only the active noise control system but also many other applications, a more detail analysis of the impulse response for the inverse notch filter needs to be required.

In this paper, we derive an explicit form for the infinite impulse response of the inverse notch filter. Additionally, we derive an explicit form for the sum of the squared impulse response. Then, we reveal the limit values of these two infinite sequences. Next, based on the analysis results, we propose a normalized notch filter whose pass band area is adjusted to unit. The normalized inverse notch filter is efficient to estimate the output power in the short time such as the frame processing. Finally, as an application of the normalized inverse notch filter, we present an $F_{0}$ estimation method for a musical sound. In the $F_{0}$ estimation method, we use multiple normalized inverse notch filters whose pass frequencies are identical to $F_{0} s$ for each monophonic sound, respectively. These normalized inverse notch filters are connected in parallel. In the estimation procedure, we detect $F_{0}$ from the inverse notch filter whose output power is largest among all the inverse notch filter output powers. From the simulation results, we see that the proposed $F_{0}$ estimation method can effectively detect the $F_{0}$ both of for the monophonic sounds and the polyphonic sounds.

\section{Performance Analysis of Inverse Notch Filter}

In this section, we explain both of the notch filter and the inverse notch filter, where the latter filter has an inverse characteristic of the notch filter. The notch filter passes all frequencies expect of the narrow frequency band centered on the notch frequency. The stop bandwidth and the 
notch frequency can be individually designed [1-7]. The several structures of the notch filter have been proposed and all of them can be transformed to the inverse notch filter. In this paper, we use the structure of the notch filter proposed in [3-5], since the inverse notch filter can be simply obtained from the notch filter's transfer function. The transfer function of the notch filter $N(z)$ is given by

$$
N(z)=\frac{1}{2}\left(1+\frac{r+\alpha z^{-1}+z^{-2}}{1+\alpha z^{-1}+r z^{-2}}\right),
$$

where $\alpha$ is a parameter to design the notch frequency and $r(-1<r<1)$ is the stop bandwidth parameter. The notch frequency parameter is given by

$$
\alpha=-(1+r) \cos \left(2 \pi \frac{F}{F_{S}}\right),
$$

where $F[\mathrm{~Hz}]$ denotes the notch frequency and $F_{S}[\mathrm{~Hz}]$ denotes the sampling frequency. When we put the stop bandwidth as $K[\mathrm{~Hz}]$, the relational expression of $r$ and $K$ is represented as

$$
r=\frac{1+\cos \left(2 \pi K / F_{S}\right)-\sin \left(2 \pi K / F_{S}\right)}{1+\cos \left(2 \pi K / F_{S}\right)+\sin \left(2 \pi K / F_{S}\right)} .
$$

From (1), we can derive the inverse notch filter represented as

$$
I(z)=1-N(z)=\frac{1-r}{2} \frac{1-z^{-2}}{1+\alpha z^{-1}+r z^{-2}},
$$

where the $I(z)$ is the transfer function of the inverse notch filter. We see from (4) that the inverse notch filter is very easy to implement. Note that the pass bandwidth parameter $r$ is also given as (3), where $K$ denotes the pass bandwidth. Figure 1 shows the structure of the inverse notch filter, where $x(n)$ is the input signal, $y(n)$ is the output signal, and $u(n)$ is the signal obtained from the IIR unit within the inverse notch filter. We see from this figure that the inverse notch filter requires only three multiplications and three additions to calculate the output signal. Figure 2 shows the frequency amplitude response of $I(z)$ when $\alpha=0\left(F=F_{S} / 2\right)$ with $r=0.8,0.9,0.99$, where the vertical axis denotes the amplitude and the horizontal axis denotes the normalized frequency. We see from Figure 2 that the amplitude at the notch frequency is 1 regardless of $r$, and the pass bandwidth becomes narrow with increasing $r$

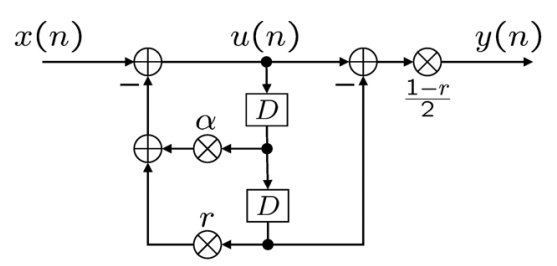

Figure 1. Structure of inverse notch filter.

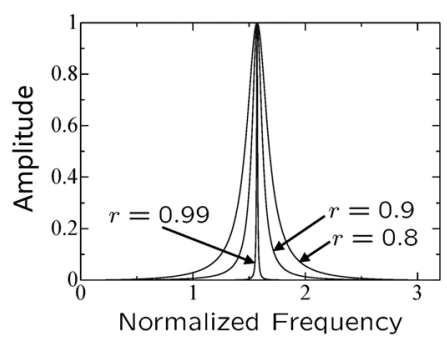

Figure 2. Power spectrum of the inverse notch filter.

toward to 1 , i.e., we can accurately extract a single sinusoidal signal by setting $r$ extremely close to 1 .

When filtering an input signal, one of the most important factors is the impulse response of the filter. We firstly derive the impulse response of the inverse notch filter as an explicit formulation. We see from (2) or Figure 1 that the signal $y(n)$ and $u(n)$ are given as

$$
y(n)=\frac{1-r}{2}(u(n)-u(n-2))
$$

with

$$
u(n)=x(n)-\alpha u(n-1)-r u(n-2) .
$$

To obtain the impulse response, we put the input signal as the impulse signal represented as

$$
x(n)=\delta(n),
$$

where $\delta(n)$ is the Kronecker's delta. In this case, (6) can be represented as the following equation

$$
u(n)+\alpha u(n-1)+r u(n-2)=0,
$$

where $n \geq 2$. Solving the above homogeneous equation with respect to $u(n)$ and introducing the initial condition that $u(0)=1$ and $u(1)=\alpha$, we obtain the solution expressed as

$$
\begin{gathered}
u(n)=\frac{2}{p} r^{(n+1) / 2} \sin (\theta(n+1)), \\
p=\sqrt{4 r-\alpha^{2}} \\
\theta=\arctan \left(\frac{p}{-\alpha}\right)
\end{gathered}
$$

We assume that $\alpha^{2}<4 r$. Note that this assumption is satisfied when $r \approx 1$. By substituting (9) into (5), we obtain the impulse response of the inverse notch filter $h(n)(n \geq 2)$ expressed as

$$
\begin{aligned}
& \left.y(n)\right|_{x(n)=\delta(n)}=h(n) \\
& =\frac{1-r}{p} r^{(n-1) / 2}\{r \sin (\theta n+\theta)-\sin (\theta n-\theta)\} .
\end{aligned}
$$

From (12), we see that the impulse response becomes close to 0 with increasing $n$ due to the term $r^{(n-1) / 2}$. When $u(n)=0,(n<0)$, we also have $h(0)$ and $h(1)$ 
represented as

$$
\begin{aligned}
& h(0)=\frac{1-r}{2}, \\
& h(1)=-\alpha \frac{1-r}{2} .
\end{aligned}
$$

Next, we formulate the sum of squared impulse response to evaluate its convergence property. Taking square of (12), the squared impulse response $h^{2}(n)(n \geq 2)$ is obtained as

$$
\begin{aligned}
h^{2}(n)= & \frac{(1-r)^{2}\left\{(1-r)^{2}+p^{2}\right\}}{2 p^{2}} r^{n-1} \\
& -\operatorname{Re}\left[\frac{(1-r)^{2}\left(1-r \mathrm{e}^{j 2 \theta}\right)^{2}}{2 p^{2}} r^{n-1} \mathrm{e}^{j 2 \theta(n-1)}\right],
\end{aligned}
$$

where we use the following relation

$$
\cos (2 \theta)=1-p^{2} / 2 r \text {. }
$$

The above relation is derived from (10) and (11). Using (13), (14), and (15), the sum of the squared impulse response $J(n)$ is represented as

$$
\begin{aligned}
& J(n)=\frac{(1-r)^{2}}{4}+\frac{(1-r)^{2} \alpha^{2}}{4}+\sum_{m=2}^{n} h^{2}(m) \\
& =\frac{1-r}{2}\left[1-\frac{r^{n}}{p^{2}}\{q-c(n)\}\right],
\end{aligned}
$$

where

$$
\begin{gathered}
q=(1+r)^{2}-\alpha^{2}, \\
c(n)=(1-r)\{\cos (2 \theta n)-r \cos (2 \theta(n+1))\} .
\end{gathered}
$$

From (17), we easily obtain the limit value of $J(n)$ with $n \rightarrow \infty$ as

$$
\lim _{n \rightarrow \infty} J(n)=\frac{1-r}{2} .
$$

The sum of the squared impulse response $J(n)$ converges to the constant which is depending on the pass bandwidth parameter $r$. From Parseval's theorem, we see that (20) is identical to the sum of the squared frequency response. Note that (20) also shows the pass band area of the inverse notch filter, since its frequency responses are almost zero expect of the pass band. Figure 3 shows the actual convergence properties for the sum of the squared impulse response with $r=0.8,0.9,0.99$, where the solid line denotes the sum of the squared impulse response and the dashed line denotes the theoretical limit calculated from (20). The horizontal axis denotes sample number. We see from Figure 3 that the sum of the squared impulse response converged to each theo- retical limit. Also we see that convergence speed becomes fast with decreasing $r$.

In the audio signal processing, the inverse notch filter is often utilized for measuring a narrowband frequency power which is corresponding to the inverse notch filter's output power. However, the pass band area of the inverse notch filter depends on the parameter $r$ as shown in (20), and thus the output power also depends on $r$. Hence, it is difficult to evaluate the inverse notch filter's output power when there exist multiple inverse notch filters which have different $r \mathrm{~s}$. To solve this problem, we derive a normalized inverse notch filter whose output power is fairly available independently with $r$. Since the output power is actually calculated in a short frame length, we have to establish the normalized inverse notch filter by taking into account the frame length. The sum of the squared output signal is given by

$$
\begin{aligned}
& V(L)=\sum_{n=1}^{L-1} y^{2}(n) \\
& =\sum_{n=0}^{L-1} \sum_{m=0}^{n} \sum_{l=0}^{n} h(m) h(l) x(n-m) x(n-l),
\end{aligned}
$$

where $L$ is the frame length. Here, we consider the case that the observed signal $x(n)$ is a white noise whose mean value and variance are 0 and $\sigma_{N}^{2}$, respecttively. Taking the expectation value of (21), we have

$$
E[V(L)]=\sigma_{N}^{2} \sum_{n=0}^{L-1} \sum_{m=0}^{n} h^{2}(m)=\sigma_{N}^{2} \sum_{n=0}^{L-1} \sum_{m=0}^{n} J(n) .
$$

Substituting (17) into (22) gives

$$
\begin{aligned}
E[V(L)] & =\sigma_{N}^{2}(1-r)^{2} / 4 \times\left[\frac{2(L-1-r)}{1-r}\right. \\
& \left.+\alpha^{2}-\frac{2 r^{2}}{p^{2}}\left\{\frac{1-r^{L-2}}{(1-r)^{2}} q-d_{L}\right\}\right],
\end{aligned}
$$

where

$$
d_{L}=\cos (4 \theta)-r^{L-2} \cos (2 \theta L) .
$$

The white noise has the same magnitude for all frequencies. Thus, it is desirable that the sum of the squared

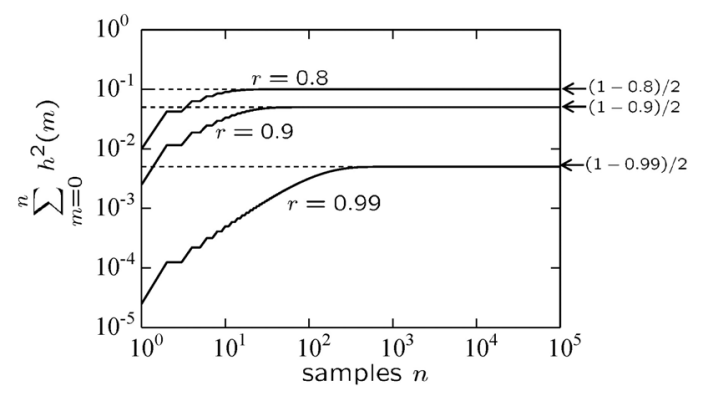

Figure 3. Convergence property for sum of squared impulse response. 
output signal of the inverse notch filter is always constant regardless of the values of $\alpha, r, L$. However, as shown in (23), the expectation value of $V(L)$ strongly depends on the respective values.

To solve this problem, we propose the following normalized inverse notch filter.

$$
\bar{I}(z)=I(z) / \sqrt{E[V(L)]}=\frac{1-r}{2 \sqrt{E[V(L)]}} \frac{1-z^{-2}}{1+\alpha z^{-1}+r z^{-2}} .
$$

The above normalized inverse notch filter adjusts the total pass band area in $L$ samples to unit. Figure 4 shows the structure of the normalized inverse notch filter, where $\bar{y}(n)$ denotes its output signal. Comparing Figure 4 with Figure 1, we see that the difference is only one multiplier's value. Hence, the computational complexities of those filters are the same.

To confirm the property of the normalized inverse notch filter, we carried out a simulation. In this simulation, the capability of the normalized inverse notch filter was compared with the general inverse notch filter shown in (4). We prepared four filters which designed by different parameters. The parameter setting is summarized in Table 1. We used white noise as the observed signal, where its mean and variance are 0 and $\sigma_{N}^{2}=1$. Figure 5 shows $E[V(L)]$ for frame length $L$, where " $\times$ " denotes the average value of $V(L)$ in 1000 simulations and the solid line denotes the theoretical value calculated by (23). In this figure, the horizontal axis de-

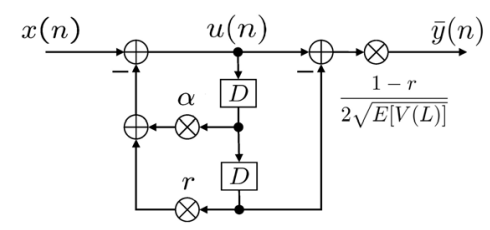

Figure 4. Structure of normalized inverse notch filter.

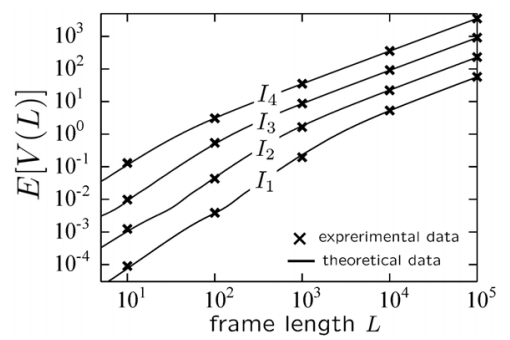

Figure 5. Sum of squared output signal of inverse notch filter output.

Table 1. Parameters for normalized inverse notch filter.

\begin{tabular}{ccccccc}
\hline Filters & & $I_{1}, \bar{I}_{1}$ & $I_{2}, \bar{I}_{2}$ & $I_{3}, \bar{I}_{3}$ & $I_{4}, \bar{I}_{4}$ \\
\hline \multirow{2}{*}{ Parameters } & $\alpha$ & -1.999 & -1.989 & -1.876 & -0.488 \\
& $r$ & 0.998 & 0.995 & 0.982 & 0.929 \\
\hline
\end{tabular}

notes frame length. We see that the each inverse notch filter $I_{m}$ gave different curves of $E[V(L)]$ due to the different parameter setting. In this case, it is not easy to evaluate the relation between filter output powers. Figure 6 shows the result of the normalized inverse notch filter $\bar{I}_{m}$. We see that all the obtained $E[V(L)]$ are unit for every frame length. Hence, we can evaluate the relation between the output power regardless of the values of $\alpha, r, L$.

\section{Application to $\boldsymbol{F}_{\mathbf{0}}$ Estimation}

In this section, as an application of the normalized inverse notch filter, we present an $F_{0}$ estimation method for musical signal. Here, we assume that the music signal consists of the $F_{0}$ frequency and its harmonics, and the amplitude of $F_{0}$ frequency is greater than other frequency amplitudes. We represent the $F_{0}$ of the music signal such as $P_{i j}$, where $i$ denotes an octave number and $j$ denotes a pitch name number, e.g., the pitch $440 \mathrm{~Hz}$ is represented as $P_{4,10}$. The estimating pitch range is set to $P_{3,9}-P_{5,3}$, where a piano sound in this frequency range has the maximum amplitude at its $F_{0}$ frequency. We set the notch frequency of the normalized inverse notch filter to correspond to the pitch $P_{i j}$. Then, the $(i, j)$-th normalized inverse notch filter is represented as

$$
\begin{gathered}
\bar{I}_{i j}(z)=\frac{1-r_{i j}}{2 \sqrt{E[V(L)]}} \frac{1-z^{-2}}{1+\alpha_{i j} z^{-1}+r_{i j} z^{-2}}, \\
\alpha_{i j}=-\left(1+r_{i j}\right) \cos \left(2 \pi P_{i j} / F_{S}\right),
\end{gathered}
$$

where $\alpha_{i j}$ and $r_{i j}$ are the notch frequency parameter and the pass bandwidth parameter for $I_{i j}(z)$, respectively. To eliminate overlap with the neighborhood pass bandwidth of $\bar{I}_{i j}(z)$, we design the pass bandwidth parameter $r_{i j}$ as

$$
\begin{aligned}
& r_{i j}=\frac{1+\cos \left(2 \pi K_{i j} / F_{S}\right)-\sin \left(2 \pi K_{i j} / F_{S}\right)}{1+\cos \left(2 \pi K_{i j} / F_{S}\right)+\sin \left(2 \pi K_{i j} / F_{S}\right)}, \\
& K_{i j}=\left\{\begin{array}{l}
P_{i j}-P_{i-1,12}, j=1 \\
P_{i, j}-P_{i, j-1}, \text { otherwise }
\end{array}\right.
\end{aligned}
$$

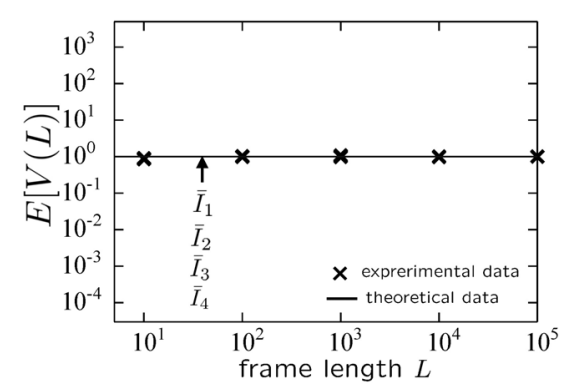

Figure 6. Sum of squared output signal of normalized inverse notch filter. 
Here, we designed the pass bandwidth as the range from its notch frequency to one of the lower neighboring notch frequency. The proposed $F_{0}$ estimator is achieved by connecting the designed normalized inverse notch filters $\left\{\bar{I}_{i j}(z)\right\}$ in parallel. The $F_{0}$ estimator is shown in Figure 7, where $\bar{y}_{i j}(n)$ denotes the output signal of $\bar{I}_{i j}(z)$. The estimation procedure is the follows: First, we calculate $V(L)$ defined in (21) for each $\bar{I}_{i j}(z)$. We then detect the normalized inverse notch filter whose $V(L)$ is largest among all of filters. Its filter number $i$ and $j$ directly gives the first $F_{0}$ estimate as $P_{i j}$. Next, we remove the normalized inverse notch filter for $P_{k i, j}(k \in \mathrm{N})$ which is corresponding to harmonics of $P_{i j}$. Repeating the above estimation procedure gives the second and latter $F_{0}$ estimates. The repetition of the estimation process is finished when all the residual $V(L) \mathrm{s}$ are smaller than the threshold.

We carried out simulations to confirm the capability of the proposed $F_{0}$ estimator. In the simulations, we set the sampling frequency $F_{S}=10[\mathrm{kHz}]$, and the frame length $L=100(=10[\mathrm{~ms}])$. We empirically set the threshold $T$ to $2 \times 10^{9}$. As the first simulation, we carried out the $F_{0}$ estimation for the monophonic sound which was played with a electronic piano. Figure 8 shows the waveform of the input signal and the estimation result, where the true octave number $i$ and pitch number $j$ are displayed on the waveform as " $i, j$ ". We plotted the

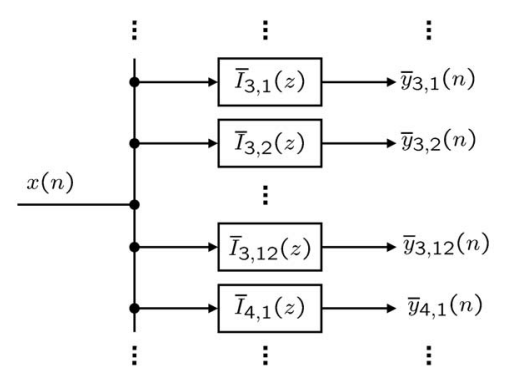

Figure 7. Structure of $F_{0}$ estimation method.

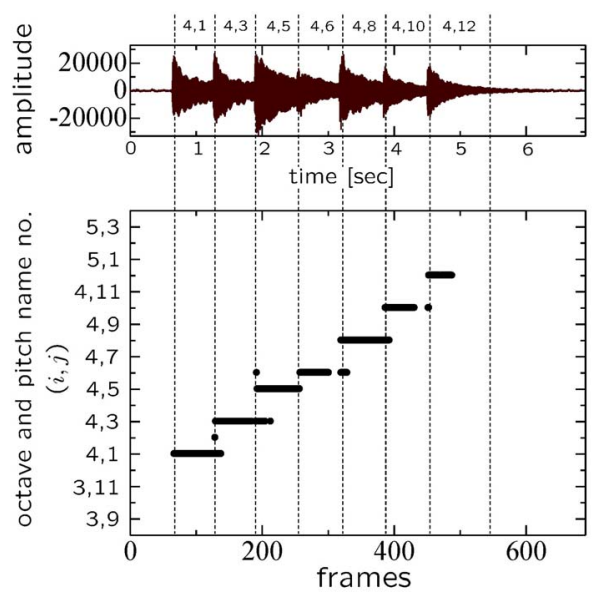

Figure 8. Simulation result for monophonic sound. estimated $F_{0}$ as the thick black line. From the result, we see that the $F_{0}$ estimation method can accurately estimate the $F_{0}$ of the observed signal, although some errors occurred in the keystroke. Additionally, we carried out the simulation for the same monophonic signal with a white noise. The estimation result shows in Figure 9. We see from the figure that the $F_{0}$ estimation method can robustly estimate the $F_{0}$ under the noisy environment as accurately as under the environment without noise.

As the second simulation, we carried out the $F_{0}$ estimation for the polyphonic sound. The polyphonic sound contains the octave note $\left\{P_{4.1}, P_{5,1}\right\}$ and $\left\{P_{4.3}, P_{5,3}\right\}$ which are known as a difficult combination to separately detect. Figure 10 shows the estimation result. We see from the figure that the $F_{0}$ estimation method can estimate the $F_{0}$ although there also exist some errors at the keystroke. Especially, the proposed method can detect $F_{0}$ for the octave note. From these results, we confirmed the normalized inverse notch filter is efficiently for $F_{0}$ estimation.

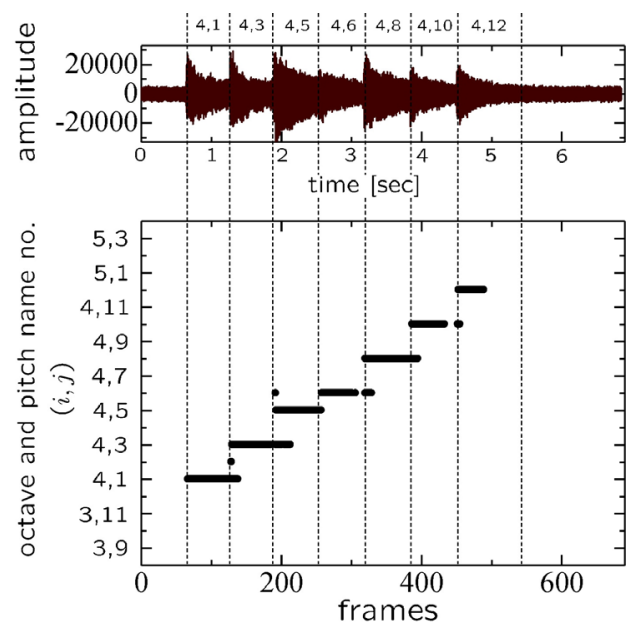

Figure 9. Simulation result for noisy monophonic sound.

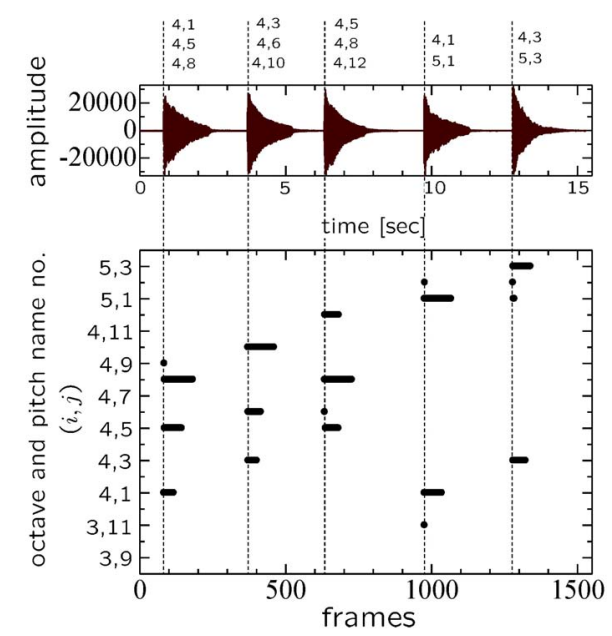

Figure 10. Simulation result for polyphonic sound. 


\section{Conclusion}

In this paper, we analyzed the inverse notch filter and derived the explicit forms for the impulse response and the sum of squared impulse response. Based on the analysis result, we derived a normalized inverse notch filter whose pass band area is identical to unit to evaluate the output powers between the multiple inverse notch filters which have different parameters. Moreover, we established an $F_{0}$ estimator by connecting the normalized inverse notch filters in parallel. Estimation results showed that the proposed $F_{0}$ estimator effectively detects $F_{0} s$ for electronic piano sound in a mid-range.

\section{REFERENCES}

[1] A. Nehorai, "A Minimal Parameter Adaptive Notch Filter with Constrained Poles and Zeros," IEEE Transactions on Acoustics, Speech and Signal Processing, Vol. 33, No. 4, 1985, pp. 983-996. doi:10.1109/TASSP.1985.1164643

[2] H. C. Chong and U. L. Sang, "Adaptive Line Enhancement by Using an IIR Lattice Notch Filter," IEEE Transactions on Acoustics, Speech and Signal Processing, Vol. 37, No. 4, 1989, pp. 585-589. doi:10.1109/29.17543

[3] C. C. Tseng and S. C. Pei, "IIR Multiple Notch Filter
Design Base on Allpass Filter," IEEE Transactions on Circuits and Systems II: Analog and Digital Signal Processing, Vol. 44, No. 2, 1997, pp. 133-136.

[4] S. C. Pei, W. S. Lu and C. C. Tseng, "Analytical TwoDimensional IIR Notch Filter Design Using Outer Product Expansion," IEEE Transactions on Circuits and Systems II: Analog and Digital Signal Processing, Vol. 44, No. 9, 1997, pp. 765-768.

[5] Y. V. Joshi and S. C. D. Roy, "Design of IIR Multiple Notch Filters Based on All-Pass Filters," IEEE Transactions on Circuits and Systems II: Analog and Digital Signal Processing, Vol. 46, No. 2, 1999, pp. 134-138.

[6] M. Goto, “A Real-Time Music-Scene-Description System: Predominant- $F_{0}$ Estimation for Detecting Melody and Bass Lines in Real-World Audio Signals," Speech Communication, Vol. 43, No. 4, 2004, pp. 311-329. doi:10.1016/j.specom.2004.07.001

[7] V. DeBrunner, “An Adaptive, High-Order, Notch Filter Using All Pass Sections," IEEE International Conference on Acoustics, Speech and Signal Processing, Vol. 3, Seattle, 12-15 May 1998, pp. 1477-1480.

[8] Y. Xiao, "A New Efficient Narrowband Active Noise Control System and Its Performance Analysis," IEEE Transactions on Audio, Speech, and Language Processing, Vol. 19, No. 7, 2011, pp. 1865-1874. doi:10.1109/TASL. 2010.2101598 